\title{
PLANETARY TRANSITS TOWARD THE GALACTIC BULGE
}

\author{
B. Scott Gaudi \\ Department of Astronomy, Ohio State University, 140 West 18th Avenue, Columbus, OH 43210; \\ gaudi@astronomy.ohio-state.edu \\ Received 2000 April 24; accepted 2000 June 29; published 2000 August 3
}

\begin{abstract}
One of the primary difficulties with using transits to discover extrasolar planets is the low probability a planet has of transiting its parent star. One way of overcoming this difficulty is to search for transits in dense stellar fields, such as the Galactic bulge. Here I estimate the number of planets that might be detected from a monitoring campaign toward the bulge. A campaign lasting 10 nights on a $10 \mathrm{~m}$ telescope (assuming $8 \mathrm{hr}$ of observations per night and a $5^{\prime} \times 5^{\prime}$ field of view) would detect five to 50 planets with radius $R_{p}=1.5 R_{\mathrm{J}}$ or two to 15 planets with $R_{p}=1.0 R_{\mathrm{J}}$, if the frequency of planets in the bulge is similar to that in the solar neighborhood. The precise number detected depends sensitively on the distribution of planets around their parent stars. Most of these planets will be discovered around stars just below the turnoff, i.e., slightly evolved G dwarfs. Campaigns involving 1 or $4 \mathrm{~m}$ class telescopes are unlikely to discover any planets, unless there exists a substantial population of companions with $R_{p}>1.5 R_{\mathrm{J}}$.
\end{abstract}

Subject headings: binaries: eclipsing — planetary systems — techniques: photometric

\section{INTRODUCTION}

The search for extrasolar planets has garnered enormous attention in recent years, due primarily to the successful implementation of radial velocity searches (Mayor \& Queloz 1995; Marcy \& Butler 1996). These searches have led to the discovery of a population of massive, close-in planets with orbital separations of $a \lesssim 0.1$ AU. Recently, it was discovered that one such planet, the companion to HD 209458, also transits its parent star (Charbonneau et al. 2000; Henry et al. 2000), yielding a measurement of the mass, radius, and density of the companion.

Despite this success, the discovery of an extrasolar planet using transits has remained elusive. There are two primary difficulties with detecting planets with transits. First, the photometric requirements ( $\leqslant 1 \%$ photometry) are quite stringent, and second, the probability that a planet will transit its parent is small ( $\$ 10 \%$ for separations $>0.05 \mathrm{AU}$ ). One way of overcoming the small transit probability is to monitor eclipsing binary stars for which the orbital plane is known to be (nearly) perpendicular to the sky (Deeg et al. 1998). Another way is to simply monitor many stars simultaneously. This can be done by employing a camera with a large field of view (VULCAN, ${ }^{1}$ Borucki et al. 1999; STARE, Brown \& Charbonneau 2000), or by monitoring very dense stellar fields. Here I focus on the latter possibility. Specifically, I determine the number of planets that might be detected in a campaign monitoring stars toward the Galactic bulge.

The motivation for this study is twofold: to determine the number of planets that might be detected via a transit search toward the Galactic bulge, and to investigate the potential of current microlensing surveys for the serendipitous detection of planets.

\section{FORMALISM}

The flux of a star being occulted by a planet is given by

$$
F(t)=F_{0}[1-\delta(t)]+F_{b},
$$

\footnotetext{
${ }^{1}$ See http://web99.arc.nasa.gov/mars/vulcan/.
}

where $F_{0}$ is the unocculted flux of the star, $F_{b}$ is the total flux from any unrelated sources, and $\delta(t)$ is the fractional deviation of the flux due to the transit, which depends on the radius of the planet relative to the star, the inclination angle $i$, and the limb darkening of the star (Sackett 1999). For a small planet $\left(R_{p} \ll R_{*}\right)$ and no limb darkening, $\delta=\left(R_{p} / R_{*}\right)^{2} \Theta(1-\tau)$, where $\Theta(x)$ is the step function, and $\tau$ is a normalized time $\tau \equiv\left(t-t_{0}\right) / t_{T}$. Here $t_{0}$ is the time of the midpoint of the transit, and $t_{T}$ is one-half the transit duration, which for circular orbits is

$$
t_{T}=\frac{P}{2 \pi} \arcsin \left[\sqrt{\left(\frac{R_{*}+R_{p}}{a}\right)^{2}-\cos ^{2} i}\right] .
$$

In reality, $\delta$ depends very sensitively on $R_{p}$ and $\cos i$ and less so on the limb darkening. I will therefore use the explicit form for $\delta$ given in Sackett (1999), but assume no limb darkening.

Since the proposed search for planets will be carried out in dense stellar fields and transits produce time-dependent variations in the flux of the stars, the data will likely be reduced with image-subtraction techniques (Tomaney \& Crotts 1996; Alard \& Lupton 1998). With image-subtraction, one measures only the time-variable portion of the flux, $\tilde{F}(t)=F_{0}[-\delta(t)]$.

\section{DETECTION PROBABILITY}

There are three requirements to detect a planet of separation $a$ and radius $R_{p}$ around a star of mass $M$, radius $R_{*}$, and flux $F_{0}$. These are: (1) the planet must transit the star, (2) at least two transits must occur during the time when observations are made, and (3) the transit must cause a detectable deviation in the light curve. If the duration of the transit is much smaller than the window of observations, then these three requirements can be considered independent.

For a planet to transit its parent star, it must have an inclination angle $\cos i \leq \cos i_{\min } \equiv\left(R_{*}+R_{p}\right) / a$. The probability 
$P_{T}$ that a planet will transit its parent star is therefore

$$
P_{T}=\frac{\int_{0}^{\cos i_{\min }} d(\cos i)}{\int_{0}^{1} d(\cos i)}=\frac{R_{*}+R_{p}}{a} .
$$

Consider a campaign lasting $N$ nights with $T_{W}$ hours per night. Defining $t=0$ as the beginning of the first night, then the times when observations are possible on (integer) night $n$ satisfy $\mathcal{T}(t)=T_{W} / 2-\left|t-n \lambda-T_{W} / 2\right| \geq 0$, where $\lambda=1$ day. Both the first transit occurring at time $t_{1}$ and the second transit at time $t_{2}$ must satisfy this relation on some (integer) nights $n_{1}$ and $n_{2}$. Note $n_{1} \geq n_{2}$. The time $t_{2}$ is given by $t_{2}=t_{1}+n_{P} P$, where $n_{P}$ is the number of periods between $t_{1}$ and $t_{2}$. Since $t_{1}$ can occur anywhere in the time span $0 \leq t_{1} \leq P$, then the probability that both transits will occur during the window(s) of observations is

$$
P_{W}=\frac{1}{P} \int_{0}^{P} d t_{1} \Theta\left[\mathcal{T}\left(t_{1}\right)\right] \Theta\left[\mathcal{T}\left(t_{1}+n_{P} P\right)\right]
$$

for any combination of $n_{1}=0,1, \ldots, N-1, n_{2}=n_{1}, n_{1}+$ $1, \ldots ., N-1$, and $n_{P}=1,2, \ldots, N \lambda / P$.

Finally, consider a transit of duration $2 t_{T}$ that occurs well within the observing window. Assuming that the transit is monitored continuously with a telescope that records $\alpha$ electrons per second per unit flux, the total signal-to-noise ratio of the transit is

$$
Q=\left(2 \alpha t_{T}\right)^{1 / 2} \frac{F_{0}}{\left(S_{\mathrm{tot}} \Omega_{\mathrm{PSF}}+F_{0}\right)^{1 / 2}}\left(\frac{R_{p}}{R_{*}}\right)^{2} G,
$$

where $S_{\text {tot }}=S_{\text {sky }}+S_{\text {back }}$ is the total surface brightness (sky + unresolved background), $\Omega_{\mathrm{PSF}}$ is the area of the point-spread function, and the function $G$ is defined as

$$
G \equiv\left(\frac{R_{*}}{R_{p}}\right)^{2}\left\{\frac{1}{2} \int_{-1}^{1} d \tau[\delta(\tau)]^{2}\right\}^{1 / 2}
$$

and depends on $R_{p} / R_{*}$, cos $i$, and the limb darkening of the star. For $R_{p} \ll R_{*}$ and no limb darkening, $G=1$. Note that an implicit assumption in equation (5) is that $\delta \ll 1$. For a transit to be detectable, $Q$ must exceed some minimum threshold $Q_{\min }$. Integration over cos $i$ then defines the probability that a transit will satisfy the signal-to-noise ratio requirement,

$$
P_{\mathrm{S} / \mathrm{N}}=\left(\cos i_{\min }\right)^{-1} \int_{0}^{\cos i_{\min }} d(\cos i) \Theta\left(Q-Q_{\mathrm{min}}\right)
$$

The total detection probability is then just $P_{\text {tot }}=P_{T} P_{W} P_{\mathrm{S} / \mathrm{N}}$.

Consider a population of stars with luminosity function $\Phi\left(F_{0}\right)$ (in units of number per area), mass-flux relation $M\left(F_{0}\right)$, and radius-flux relation $R\left(F_{0}\right)$. Assuming a fraction $f$ of these stars have planets of radius $R_{p}$ distributed according to $F(a) \mathrm{d} a$ (which I will assume is independent of $F_{0}$ ), then the number of planets detected in a field of view of area $\Omega_{\mathrm{CCD}}$ is

$$
N_{\mathrm{det}}=f \Omega_{\mathrm{CCD}} \int d a \mathcal{F}(a) \int d F_{0} P_{\mathrm{tot}} \Phi\left(F_{0}\right)
$$

\section{APPLICATION TO THE GALACTIC BULGE}

In order to apply the results of $\S 2$ to the Galactic bulge, several assumptions must be made about the population being monitored and the telescope and observational setup. I will consider observations in the $I$ band, which provides a good compromise between dust extinction and high sky background. I construct an I-band luminosity function (LF) by combining the determination toward Baade's window by Holtzman et al. (1998) on the bright $\left(M_{I} \leq 9\right)$ end with the local M dwarf LF as determined by Gould, Bahcall, \& Flynn (1997) for the faint end. I normalize the latter to agree with Holtzman et al. (1998) at $M_{I}=7.25$. I adopt a distance modulus of 14.52 and an extinction of $A_{I}=1.0$, appropriate for Baade's window. For $M\left(F_{0}\right)$ and $R\left(F_{0}\right)$, I use the $10 \mathrm{Gyr}$, solar metallicity isochrone of Girardi et al. (2000). Varying the age and/or metallicity of the population within a reasonable range does not affect the results substantially.

I assume $S_{\text {sky }}=19.5 \mathrm{mag} \operatorname{arcsec}^{-2}$, corresponding to the sky brightness of a relatively dark site averaged over the phases of the moon. Over a 10 day observing window, the sky brightness can vary by as much as $0.5 \mathrm{mag}$ in $I$. Therefore, the signalto-noise ratio calculations presented here may be underestimates or overestimates depending on the phase of the moon. I assume $\Omega_{\mathrm{PSF}}=\pi \theta^{2}$, where $\theta$ is the seeing, and $\alpha=$ $600(D / 10 \mathrm{~m})^{2}$ electrons per second at $I=20$, where $D$ is the telescope diameter. In the crowded fields toward the Galactic bulge, the surface brightness $S_{\text {back }}$ due to unresolved sources will depend strongly on the seeing. To estimate $S_{\text {back }}$, I first use the LF to determine the magnitude at which the sources become unresolved, i.e., I determine the $I_{\min }$ such that all sources brighter than $I_{\min }$ contribute on average one star per seeing disk. Then $S_{\text {back }}$ is just the total surface brightness due to all stars fainter than $I_{\text {min }}$.

These assumptions can now be combined with the results of $\S 2$ to determine the number of planets that may be detected in a monitoring campaign toward the Galactic bulge as a function of the various input parameters. Figure 1 shows the total detection probability $P_{\text {tot }}$ for a planet of radius $R_{p}=1 R_{\mathrm{J}}$ and separation $a=0.05 \mathrm{AU}$ as a function of $I$ magnitude, assuming 10 nights of $8 \mathrm{hr}$ per night on a $10 \mathrm{~m}$ telescope with $\theta=0$.'75 and a minimum signal-to-noise ratio of $Q_{\min }=10$ for a detection. For planets orbiting stars slightly fainter than the turnoff, $19 \leq I \leq 21$, almost all transits occurring during the windows of observation create significant $\left(Q \geq Q_{\min }=\right.$ 10) transits, i.e., $P_{\mathrm{S} / \mathrm{N}} \sim 1$. For $I \leq 19$, the radii of the sources rapidly increase, rendering the transits undetectable. For $I \geq$ 21 , the sources produce too few photons to pass the signal-tonoise ratio criterion. For this separation, $a=0.05 \mathrm{AU}$, the probability $P_{W}$ that the planet will transit twice during the windows of observation drops precipitously for $20 \leq I \leq 21$, since the period of the planet, $P=4.08\left[M(I) / M_{\odot}\right]^{-1 / 2}$ days, moves into "antiresonance" with the observation window, $T_{W}=$ $8 \mathrm{hr}$. However, such effects will approximately average out when a range of separations is considered. Furthermore, my assumption of contiguous nights of equal duration is oversim- 


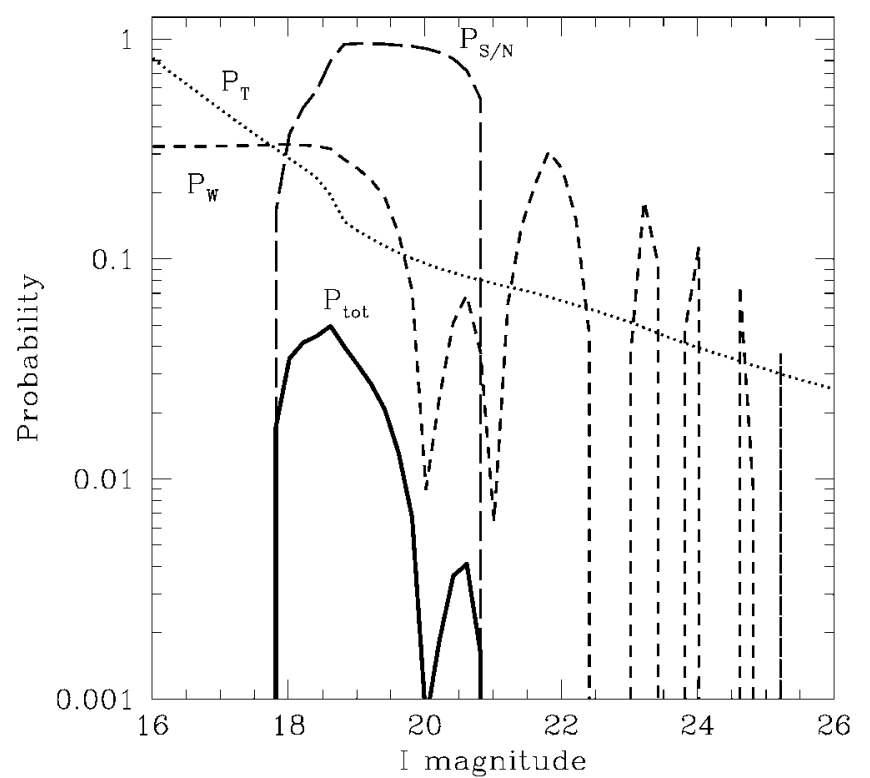

FIG. 1.-Detection probability as a function of $I$ magnitude for a planet with radius $R_{p}=1.0 R_{\mathrm{J}}$ and separation $a=0.05 \mathrm{AU}$, or period $P=$ $4.08\left[M(I) / M_{\odot}\right]^{-1 / 2}$ days, assuming 10 nights of $8 \mathrm{hr}$ per night on a $10 \mathrm{~m}$ telescope with 0 "'75 seeing. The solid curve shows the total detection probability, which is given by $P_{\text {tot }}=P_{T} P_{W} P_{\mathrm{S} / \mathrm{N}}$, where $P_{T}$ is the probability that the planet will transit its parent star, $P_{W}$ is the probability that two transits will occur during observation windows, and $P_{\mathrm{S} / \mathrm{N}}$ is the probability that the transit will have total signal-tonoise ratio $>10$.

plified: those separations that are detectable will depend on the actual spacing and duration of nights of the observations.

The number of planets detected during a monitoring campaign can now be determined by integrating over the luminosity function of the sources and the separation of the companions (see eq. [8]). This requires knowledge of the frequency and distribution of planetary companions to the bulge sources, of which little is known. However, radial velocity surveys do provide such information for planetary companions to solartype stars in the local neighborhood. Cumming, Marcy, \& Butler (1999) performed a statistical study of 74 solar-type stars from the Lick radial velocity survey. Of these, two had confirmed planetary $\left(M_{p} \leq 10 M_{\mathrm{J}}\right)$ companions with separations $\leq 0.1$ AU, i.e., $3 \% \pm 2 \%$ of the sample. Furthermore, they note that the distribution in orbital radius shows a "pileup" toward small orbital radii, but that this trend is not statistically significant. It does hint, however, that the distribution in $a$ may not be uniform. Finally, there are indications that there exists a hard cutoff in minimum separation: of the 12 planets known with $a \leq 0.1 \mathrm{AU}$, none have separations $\leqslant 0.04 \mathrm{AU}$. Since the physical processes that lead to this minimum separation are not well understood, it is not clear how they might be altered in different environments. Thus, it is not clear whether this hard cutoff would also be present for systems in the Galactic bulge. Regardless, it is important to ascertain whether planets of separation $\leq 0.04 \mathrm{AU}$ are detectable around bulge stars in order to test whether or not this minimum separation exists. I will therefore assume that $f=1 \%$ of all stars have planetary companions and adopt a uniform distribution in $\log a$ between 0.01 and $0.1 \mathrm{AU}$ for the majority of the calculations, but also consider a distribution that is uniform in $\log a$ between 0.04 and $0.15 \mathrm{AU}$.

Figure 2 shows the differential distribution of the number of detected planets per unit area, $d\left(N_{\mathrm{det}} \Omega_{\mathrm{CCD}}^{-1}\right) / d a$, as a function

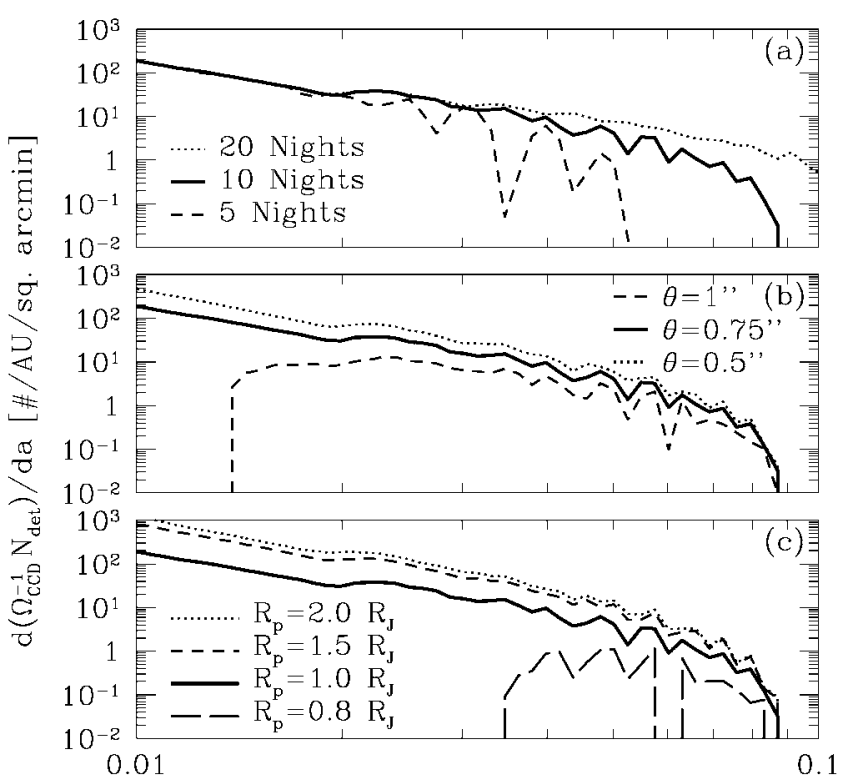

Orbital Separation (AU)

FIG. 2.-Number of planets detected per AU per square arcminute as a function of orbital separation, assuming that $1 \%$ of all stars have planets distributed uniformly in $\log a$ between $a=0.01$ and $0.1 \mathrm{AU}$. All other parameters are held constant at $R_{p}=1.0 R_{\mathrm{J}}, N=10$ nights, $T_{W}=8 \mathrm{hr}, D=$ $10 \mathrm{~m}$, and $\theta=0.75$, unless otherwise stated. (a) The effect of varying the total number of nights. ( $b$ ) The effect of varying the seeing $\theta$. (c) The effect of varying the radius of the planet $R_{p}$.

of $a$ for $R_{p}=1 R_{\mathrm{J}}$, assuming that $1 \%$ of all stars have planets distributed uniformly in $\log a$ between $a=0.01$ and $0.1 \mathrm{AU}$ and the fiducial campaign with parameters $N=10, T_{W}=8 \mathrm{hr}$, $D=10 \mathrm{~m}$, and $\theta=0.75$. Each panel shows the result of varying $N, \theta$, and $R_{p}$. For this input distribution, decreasing the duration of the campaign to $N=5$ nights will not substantially decrease the number of detections: most of the planets lost will be at large orbital separations, where the detection efficiency and frequency are already low. Similarly, doubling the number of nights will not substantially enhance the number of detections, although it enables the detection of planets at orbital separations larger than $0.1 \mathrm{AU}$. The number of detected planets depends quite crucially on the seeing: increasing the seeing increases the number of unresolved sources and therefore adds to the background flux. As $\theta$ increases, the signalto-noise ratio degrades, and transits quickly fall below the minimum detectable threshold. Thus, detections are lost and preferentially so for smaller separations (where the duration of the transits are shorter). Conversely, improving the seeing dramatically increases the number of detections. Therefore, transit searches toward the Galactic bulge should be carried out at good sites with seeing better than $1^{\prime \prime}$. The dependence of the number of detections on $R_{p}$ is similar to that of $\theta$ : as $R_{p}$ decreases, transits fall below the minimum detectable threshold, again preferentially so for smaller separations where the duration of the transits are shorter.

Figure 3 shows the number of planets detected per unit area $N_{\text {det }} \Omega_{\mathrm{CCD}}^{-1}$, as a function of $R_{p}$, for telescope apertures of $D=$ 10,4 , and $1 \mathrm{~m}$ and the fiducial parameters $N=10, T_{W}=8$ $\mathrm{hr}$, and $\theta=0^{\prime \prime} 75$. For a $5^{\prime} \times 5^{\prime}$ field of view, a $10 \mathrm{~m}$ telescope would detect $\sim 50$ planets of radius $R_{p}=1.5 R_{\mathrm{J}}$, and $\sim 15$ planets if $R_{p}=1.0 R_{\mathrm{J}}$. Most of these planets will be discovered around stars at or slightly below the turnoff: the number- 


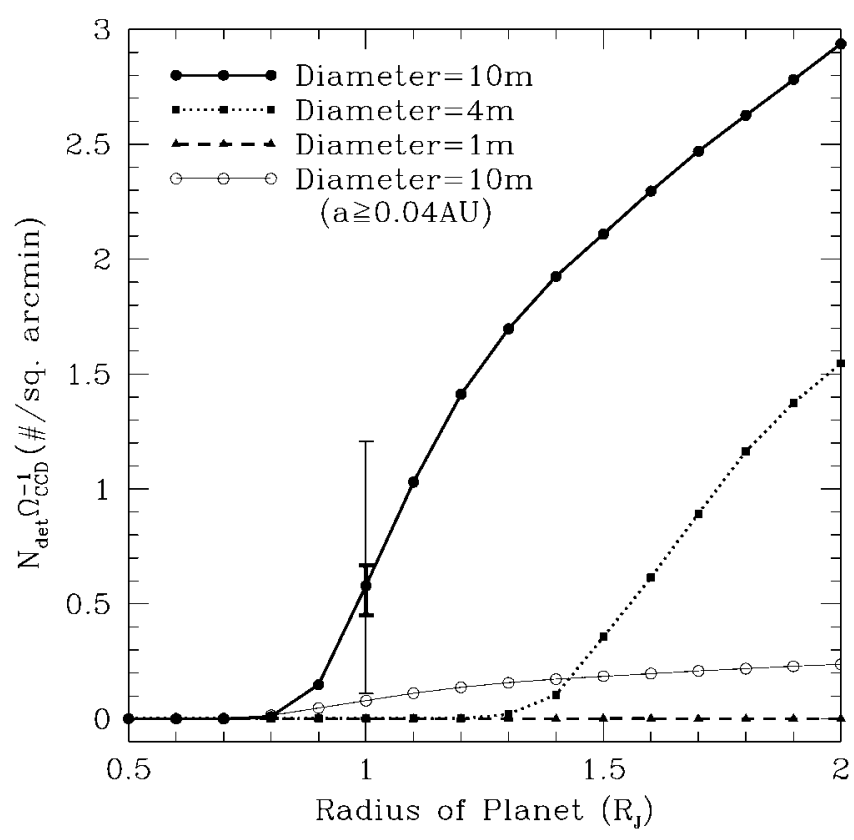

FIG. 3.-Number of planets detected square arcminute as a function of the radius of the planet for three different telescope apertures, assuming 10 nights of $8 \mathrm{hr}$ per night. I have assumed a seeing of $\theta=0.75$ and that $1 \%$ of all stars have planets distributed uniformly in $\log a$ between $a=0.01$ and 0.1 AU. The thick error bar on the point for $D=10 \mathrm{~m}$ and $R_{p}=1 R_{\mathrm{J}}$ corresponds to changing the number of nights by $N=10_{-5}^{+10}$. The thin error bar corresponds to changing the seeing by $\theta=\left[00^{\prime \prime} 75 \pm 0\right.$ "'25]. The light solid line with open circles is the number of detected planets for $D=10 \mathrm{~m}, 10$ nights, $\theta=$ 0.75 , and assuming that $1 \%$ of all stars have planets distributed uniformly in $\log a$ between $a=0.04$ and $0.15 \mathrm{AU}$.

weighted $I$ magnitude of the sources with detected planets is $\bar{I}=19.4$. For small planetary radii, $R_{p} \lesssim 0.8, N_{\mathrm{det}}<1$. Thus, if most planets have radii less than that of Jupiter, it will be quite difficult to detect them around stars in the Galactic bulge, unless the seeing is excellent, $\theta \leq 0.5$ (see Fig. 3). For 1 and $4 \mathrm{~m}$ class telescopes, the number of detected events is almost negligible below $1.5 R_{\mathrm{J}}$. Therefore, such monitoring campaigns are unlikely to detect any planets, unless there exists a substantial population of companions in the Galactic bulge with radii $R_{p}>1.5 R_{\mathrm{J}}$. Also shown is $N_{\mathrm{det}} \Omega_{\mathrm{CCD}}^{-1}$ for $D=10 \mathrm{~m}$ and the fiducial parameters, assuming that $1 \%$ of all stars have planets distributed uniformly in $\log a$ between 0.04 and $0.15 \mathrm{AU}$. For this distribution, the number of detected planets is a factor of 3-10 times lower.

\section{CONCLUSIONS}

In this Letter, I have estimated the number of planets that may be detected by transits in a monitoring campaign toward the Galactic bulge. An investment of 10 clear nights of $8 \mathrm{hr}$ per night on a $10 \mathrm{~m}$ telescope at a site with excellent $(0.75)$ median seeing would result in the detection of two to 15 planets of Jupiter size, if the frequency and distribution of planetary companions to stars in the Galactic bulge is similar to those of $\mathrm{G}$ dwarfs in the solar neighborhood. The precise number detected depends sensitively on the distribution of planets around their parent stars. Most of these planets will be found around stars slightly fainter than the turnoff, i.e., evolved G or early $\mathrm{K}$ dwarfs. An excellent site is required: if the seeing is substantially worse than 0.75 , the number of detections will be considerably smaller. Similar campaigns involving 1 or 4 $\mathrm{m}$ class telescopes are unlikely to result in any detections toward the bulge. Thus, collaborations currently monitoring the Galactic bulge for microlensing events are unlikely to serendipitously detect any transits.

I would like to thank Andreas Berlind, Andrew Gould, and Paul Martini for helpful discussions, Alberto Conti and Penny Sackett for reading the manuscript, and the anonymous referee for several suggestions that led to an improved manuscript. The original idea for this Letter is due to Penny Sackett. This work was supported by an Ohio State University Presidential Fellowship.

\section{REFERENCES}

Alard, C., \& Lupton, R. H. 1998, ApJ, 503, 325

Borucki, W. J., Caldwell, D. A., Koch, D. G., Webster, L. D., Jenkins, J. M., \& Ninkov, Z. 1999, BAAS, 195, 24.01

Brown, T. M., \& Charbonneau, D. 2000, in Disks, Planetesimals, and Planets, ed. F. Garzon \& T. J. Mahoney (San Francisco: ASP), in press (astro-ph/ 0005009)

Charbonneau, D., Brown, T. M., Latham, D. W., \& Mayor, M. 2000, ApJ, 529, L45

Cumming, A., Marcy, G., \& Butler, R. P. 1999, ApJ, 526, 890

Deeg, H. J., et al. 1998, A\&A, 338, 479

Girardi, L., Bressan, A., Bertelli, G., \& Chiosi, C. 2000, A\&AS, 141, 371
Gould, A., Bahcall, J. N., \& Flynn, C. 1997, ApJ, 482, 913

Henry, G. W., Marcy, G. W., Butler, R. P., \& Vogt, S. S. 2000, ApJ, 529, L41

Holtzman, J. A., Watson, A. M., Baum, W. A., Grillmair, C. J., Groth, E. J., Light, R. M., Lynds, R., \& O’Neil, E. J. 1998, AJ, 115, 1946

Marcy, G. W., \& Butler, R. P. 1996, ApJ, 464, L147

Mayor, M., \& Queloz, D. 1995, Nature, 378, 355

Sackett, P. D. 1999, in Planets outside the Solar System: Theory and Observations, ed. J.-M. Mariotti \& D. M. Alloin (NATO ASI Ser. C, 532; Dordrecht: Kluwer), 189

Tomaney, A. B., \& Crotts, A. P. S. 1996, AJ, 112, 2872 